Check for updates

The BMJ

Cite this as: $B M / 2021 ; 372: n 108$ http://dx.doi.org/10.1136/bmj.n108 Published: 13 January 2021

\title{
Covid-19: Many ICU staff in England report symptoms of PTSD, severe depression, or anxiety, study reports
}

\section{Elisabeth Mahase}

Nearly half of intensive care unit (ICU) and anaesthetic staff surveyed for a study reported symptoms consistent with a probable diagnosis of post-traumatic stress disorder (PTSD), severe depression, anxiety, or problem drinking. ${ }^{1}$

The preprint, produced by researchers at King's College London, aimed to get a picture of the rates of probable mental health disorders in ICU and anaesthetic staff in six English hospitals during June and July 2020. It found that while over half reported good wellbeing, many showed signs of mental health problems.

The authors said that during the covid-19 pandemic, ICU staff have "faced a particularly challenging time" because of the high mortality among ICU patients with covid-19. "Difficulty in communication and providing adequate end-of-life support to patients and their next of kin, because of visiting restrictions, has been a specific stressor for all staff working in ICU," they said.

The researchers asked volunteers to complete an anonymised survey of questions regarding depression, anxiety symptoms, symptoms of PTSD, wellbeing, and alcohol use. Just over 700 staff members completed the surveys, including 291 doctors (41\%), 344 nurses (48.5\%), and 74 other healthcare staff (10.4\%).

The preprint said that over half (58.8\%) of participants reported good wellbeing on the Warwick Edinburgh Mental Wellbeing Scale $(n=418,58.8 \%)$. However, $45.4 \%(n=322)$ met the threshold for probable clinical significance on at least one of the following measures: severe depression (6.3\%), PTSD $(39.5 \%)$, severe anxiety (11.3\%), or problem drinking $(7.2 \%)$.

The study also reported that $13.4 \%$ of respondents reported having thoughts that they would be better off dead, or of hurting themselves several days or more frequently in the two weeks before completing the survey. Nurses were more likely to report these thoughts than other healthcare staff $(19.2 \% v 7.6 \%$ for doctors and 9.5\% for clinical staff.)

The preprint said, "While further validation studies are required to better understand what proportion would meet diagnostic criteria for PTSD on clinical assessment, these data suggest that ICU clinicians are at a significantly elevated risk of suffering with PTSD.

"Our findings of high levels of PTSD, and other mental health difficulties such as depressive anxiety disorders, are highly relevant given that there is strong evidence poor mental health is associated with functional impairment which would increase the risk of patient safety incidents.”

A major limitation of the research was that there was no baseline to compare these figures with as no survey was taken before the pandemic. The authors did note that a 2015 study of 335 ICU staff reported probable PTSD at $8 \%$ among staff working with adults and $17 \%$ among staff working with children.

Speaking to The BMJ, Clare Gerada-who runs the NHS Practitioner Health Programme (PHP)-noted a number of limitations to the study, including that people who filled out the survey were more likely to have problems to report. She said, however, that the PHP, which provides help for doctors and dentists across England with mental illness and addiction problems, has seen high rates of mental health problems among doctors during the pandemic, especially for severe depression.

Meanwhile, BMA mental health lead Andrew Molodynski said the findings were "extremely troubling, but sadly not surprising."

“The BMA's most recent tracker survey revealed that almost $60 \%$ of doctors in England, Wales, and Northern Ireland are now suffering from some form of anxiety or depression, with $46 \%$ saying their condition had worsened since the start of the pandemic," he said.

"This research further highlights the vital importance of fostering a supportive workplace culture, and the need to provide universal access to high quality wellbeing support and occupational health services across the board. Equally, health and care workers must be protected at work and so it is crucial that they are urgently vaccinated so that they are able to be fit and well to continue providing care and keeping services running at time when the NHS is under unparalleled pressure."

The research was funded by the National Institute for Health Research and Public Health England. One of the researchers works for NHS England.

Greenberg N, Weston D, Hall C, Caulfield T, Williamson V, Fong K. The mental health of staff working in intensive care during covid-19.MedRxiv 2020.11.03.20208322 [Preprint]. 2020. www.medrxiv.org/content/10.1101/2020.11.03.20208322v2. 